\title{
Bio-Dynamic Rhythm Track for Daily Physical-Skills "Force Special Speed and Bearing Performance" of Components Structure of "Unso Kata" Movement Pattern as an Indicator for Training Periods' Planning for Karate
}

\author{
Ahmed Mahmoud Mohamed Ibrahim*, Mohamed Mosaad Hassan Hamed Awad**
}

\begin{abstract}
:
Wholesale kinetic "kata" essence of real karate, and one of the poles of the sport and in the right way and move on to understanding the performance skills of styles that make up the structure of structural Karate, although attention from trainers during the training the players on the "kata" Beauty kinetic be directed to perform skill components with lack of focus on the actual application of those components, as well as the legalization of individual loads directed to development and a high level of skill to achieve player or a player.
\end{abstract}

Study procedures:

\section{Methodology:}

Use descriptive survey method and the nature of the relevance and objectives of the study.

The human sphere:

sample was selected in a deliberate manner total (12) for the player, under 16 years of stage during the sports season 2010 - 2011.

Test the skills and methods of measurement of kinetic sentences:

- test and evaluate the level of some of the physical limitations - foundational skills methods structural building motor skills your wholesale "Aonso kata, Unsu kata.", Through multiple Altokiet "09:00 AM 0.11, 01:00 AM 0.3 0.5 m, 7 pm and 9 pm"

- statistical area: Use-way analysis of variance and one, the test is less difference. "LSD"

\section{Conclusions:}

The period of time that the determinants of bio-dynamic rhythm track physical - skills for "speed and performance durability" of wholesale auto players (kata) is highest during the day occurred during that time (11:00 to 13:00 AA), 5 to 7 ๗m.

skill as an indicator for planning training periods for players Wholesale Car (kata) $\varpi^{\bullet}$ can be recommended rationalization period of time and in accordance with the highest average daily biorhythms of the determinants of physical-Karate.

\section{Introduction:}

$\mathrm{T}$ he movement pattern "kata" is considered the real karate essence, and one of the poles of this sport and the right and effective way to understand skills performance of the methods constituting the karate structure, though, coaches' attention during Kata training is

\footnotetext{
* Professor of Athletic Training for Karate, Department of Combat Training and Individual Sports, Faculty of Physical Education for Boys, Alexandria University, Egypt.

** MD, Faculty of Physical Education for Boys, Alexandria University, Egypt.
}

directed to components' skill performance with lack of focus on components actual practice, as well as individual standardization of directed loads to develop and increase player's skill achievement level.

Nakayama (1981, p6) that kata in karate is the logical arrangements of punching, , stricken and in specific directions continue for fifty Kata or " official exercises " practiced in present time and some of them have been inherited from generation to generation and others has been recently constructed. 
Ahmed Mahmoud Ibrahim (2005, p27, 285 287)

argued that "Kata" is considered performing international accepted succession from defensive and offensive styles of blocking, punching, striking and kicking in different directions and differentiated conflicts directed to the three attacker's body levels or group of dummy attackers by taking different and numerous valance positions.

"kata" can be defined as coherent series of combination of motor performance, consisting of defensive and offensive methods performed y the player in internationally regulated sequence against a group of dummy competitors, in different directions, speeds and strength vary according to competitive position.

Ahmed Mahmoud Ibrahim (2005, p:284) adds that during dummy fight "kata" competitions with international movement pattern, we find that player requires participation in the competition throughout the day in same tournament "e.g. individual " including introductory, semi final and final rounds with commitment to perform internationally reglated movement pattern, in terms of motor performance sequence, offensive and defensive methods' speed and strength, as well as compound skills forming movement pattern, also added to this match load for explanation and practice of movement pattern components Albonkaa Bunkai" and that the team that reaches the final, which increases the effects of pregnancy Alambaraúa on different devices.

There in five karate schools 4 of them certified by World Karate Federation (WKF) Which are: - Shotokan, Shito-Ryu, Goji - Ryu, and Wado; each of these schools have number of movement patterns (Kata) " players perform from it as per Karate international law, and performance may be mandatory required; therefore each school have (2) compulsory katas "Shitei - Kata" determined by WKF, while the remaining Kata are optional "Touki - Kata" which the player or team can choose from it what will be performed during semi-final and final roles in the competition. All these schools with around 50 internationally classified Katas follow two karate basic styles . Each of them found and developed separately and have it is own philosophy, both in Kata performance methods and also in training methods of these and its rhythm.

The first one is Shorei Style which depends in Kata and offensive and defensive methods on streamline performance and prorated sudden movement with using narrow balance positions like "Sanchin Dachi", Shiko Dachi with using speed relatively greater than strength during this style Kata performance , Kata in this methods (e.g., and not limitesd to) are :: - " Tekki, Jion, Hangetsu, Jutte, Sochin".

The second style is Shoren Style, which depends on streamline performance with movement with rotation between speed and slowness during defensive and offensive methods' performance with availability of individual integrated skills individually in full compatibility during Kta performance, "Shoren" also characterized by achieving synchronization in terms of speed and strength during Kata components' performance which appear as special rhythmic for each Kata components. Player in this style take wide basic positions like Zenkutus Dachi, Kokut su Dashi, Kiba Dachi "and falls under this style Katas like: Kanku - Dai, Empi, Ganka ku, and Unsu, Gojushiho sho.

\section{Unsu Kata and its Charectrestics:}

Ahmed Ibrahim (2005, p193) Unsu Kata means (clouds' hands ) and have 52 styles, its performance time 90 seconds and is considered one of the most difficult Katas in terms of performance.

Unsu Kata featured characteristics

- Overall performance time: 90 seconds

- Defensive styles: 27 styles

- Offensive styles: 23 styles

Percentage of energy system prevailing during performance:

Aerobic 10\%, phosphate- anaerobic $70 \%$, lactic-anaerobic $20 \%$

Unsu Kata most important physical and psychomotor requirements

* Power Strength endurance

* Speed endurance

* skill performance endurance 
Figure (1)

Unsu Kata components structure, skill, offensive and defensive balance styles, balance positions and feet work (Ahmed Ibrahim,2005, p193)

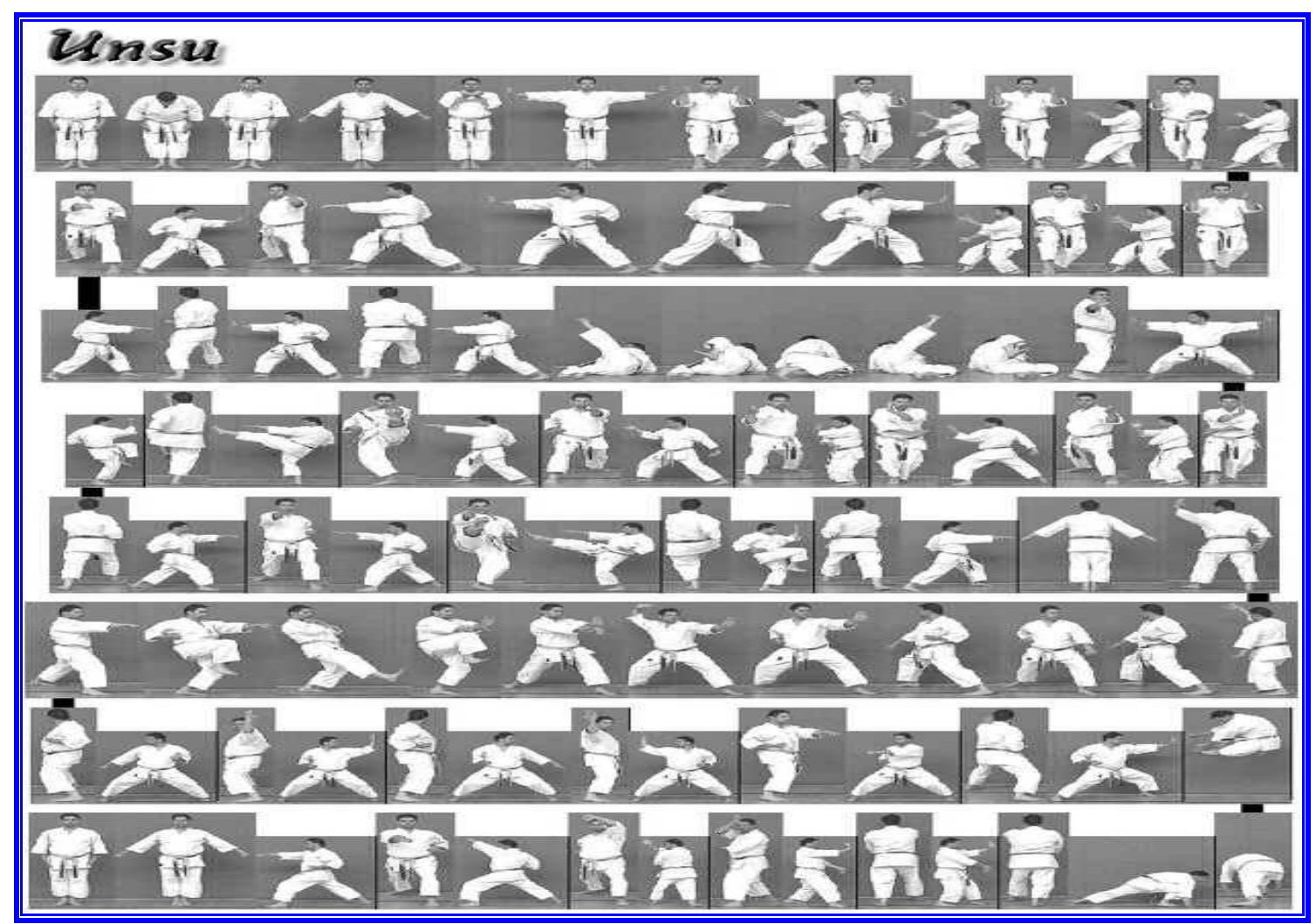

Figure (2)

Performance track of Unsu Kata components structure, skill, offensive and defensive balance styles, balance positions and feet work (Ahmed Ibrahim,2005, p193)

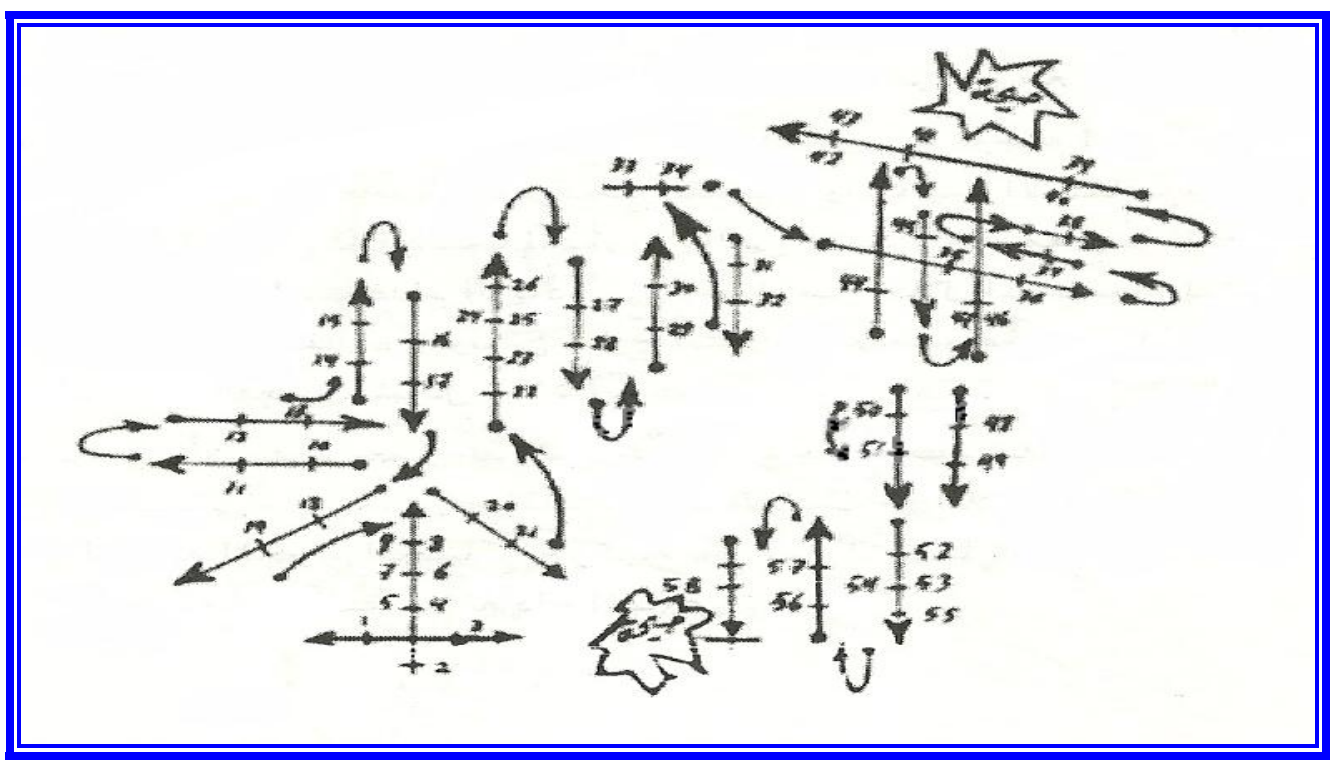

Bio- rhythm is considered one of the modern science used in physical education field as each individual's work efficiency vary according to different day times which work performed in; in the sports field we must describe bio-rhythm importance in terms of higher sports results in terms of athletic records, and performance levels during international and Olympic tournaments which make it clear that using scientific research is important to find new ways to increase sports training effectiveness and develop new trends related to specialized sport activity nature, first step to excel in specific activity is the appropriate selection for players suits it, which requires availability of special biological, physical and skill parameters, with 
scientific training programs which are considered the cornerstone of athlete making.

Aboulela Abdel Fattah and Subhi Hassanein (1997, p: 391) argues that bio-rhythm include more than 400 body functions, where is body temperature is bio-rhythm in its lowest degrees in the morning and body temperature goes up until it reaches its maximum at six o'clock pm as well as that kidney and endocrines bio-rhythm during 24-hour period shows they are more active during the first hours of the day (morning). While Sleep, wakeup, work and rest are the main bio-rhythms that all bio-rhythms of the various body systems are connected. (7: 391)

Ali ElBek and Sabri Omar (1994, p47-48) mentioned that daily bio-rhythm for functional activity of nervous and work system is playing the main role of synchronization of all processes that occur in repeated cycles as daily changes in nervous system in humans are closely related to sleep and waking cycle

From what previously mentioned it is easy to identify bio-rhythm importance and its role in influencing physical parameters which affect human body vital system' efficiency and its capabilities to work throughout the day and how important is that for physical activity in general and specially in developing new training methods. Therefore scientific studies and researches trying to identify the most important physical, skill and functional requirements suits sports activity nature and affect positively training process effectiveness to reach high athletic levels, so it is clear this study importance trying to explore the bio-rhythm dynamic track for some skill-physical parameters for Unsu kata components structure as an indicator for training periods' planning for karate.

\section{Study procedures:}

\section{Methodology:}

Descriptive approach was used as it appropriate for study nature and objectives.

\section{Human domain:}

Sample was selected intentionally totaled to (12) player registered as Kata players in Victoria club under 16 years age during sports season 2010 - 2011.

\section{Time domain:}

Main experiment performed in the period November 1st, 2010 to November 30th , 2010

\section{Kata skills measurement test:}

- Evaluation test for some physical - skills parameters for Unsu Kata components in specific times 9 and 11 am, 1, 3, 5, 7 and 9 $\mathrm{pm}$,

- Statistical work: Researcher used one way ANOVA test, and LSD test. All work done using SPSS ver. 11.

\section{Results and Discussions:}

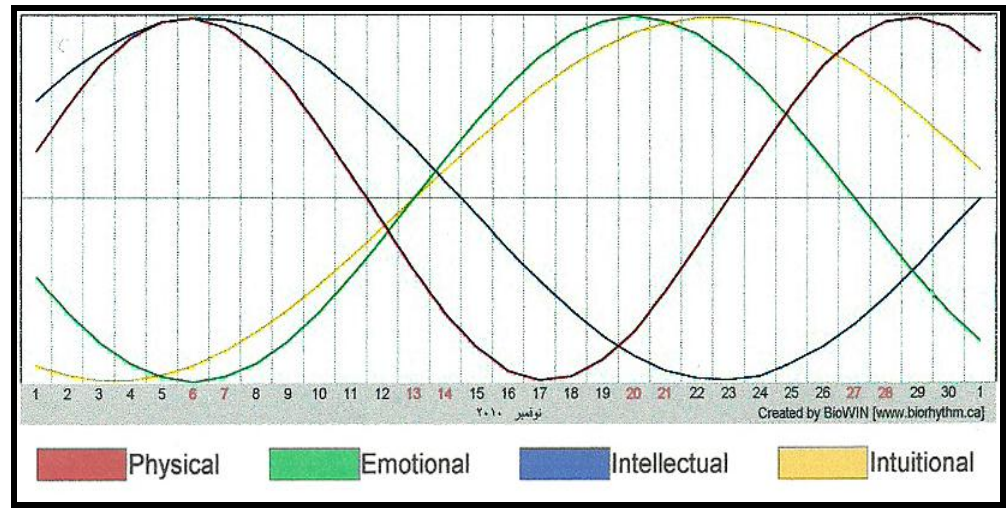


Figure (4)

Dynamic track for monthly bio- rhythm values for Kata players (No 2) during November 2010 under study

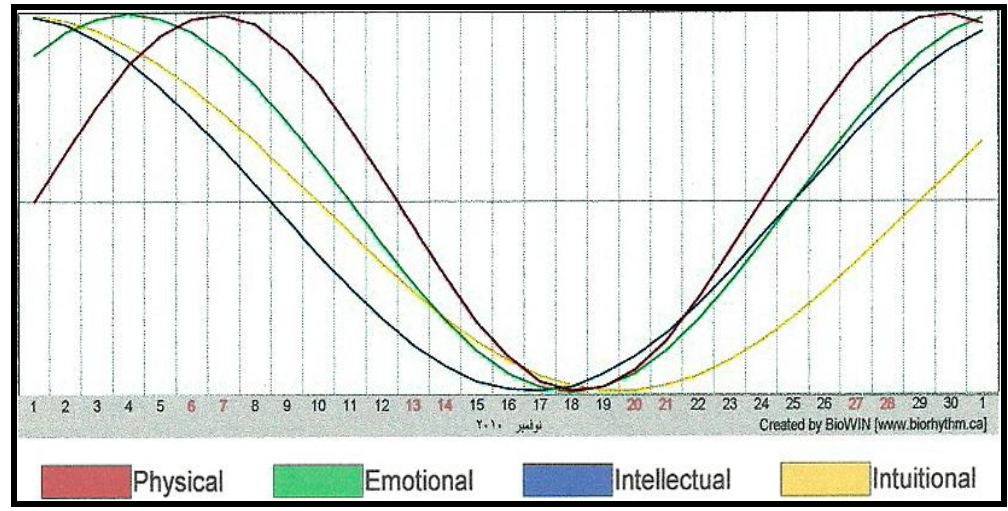

Figure (5)

Dynamic track for monthly bio- rhythm values for Kata players (No 3) during November 2010 under study

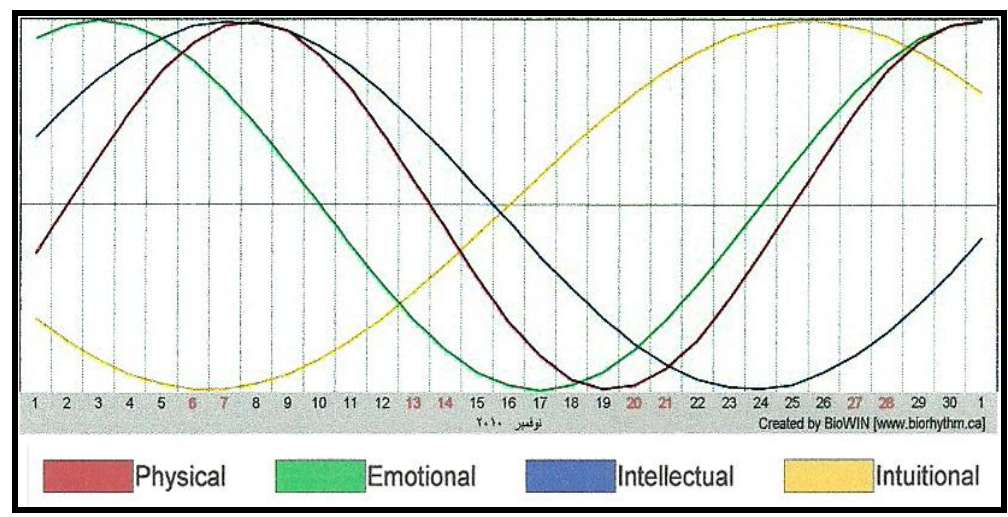

Figure (6)

Dynamic track for monthly bio- rhythm values for Kata players (No 4) during November 2010 under study

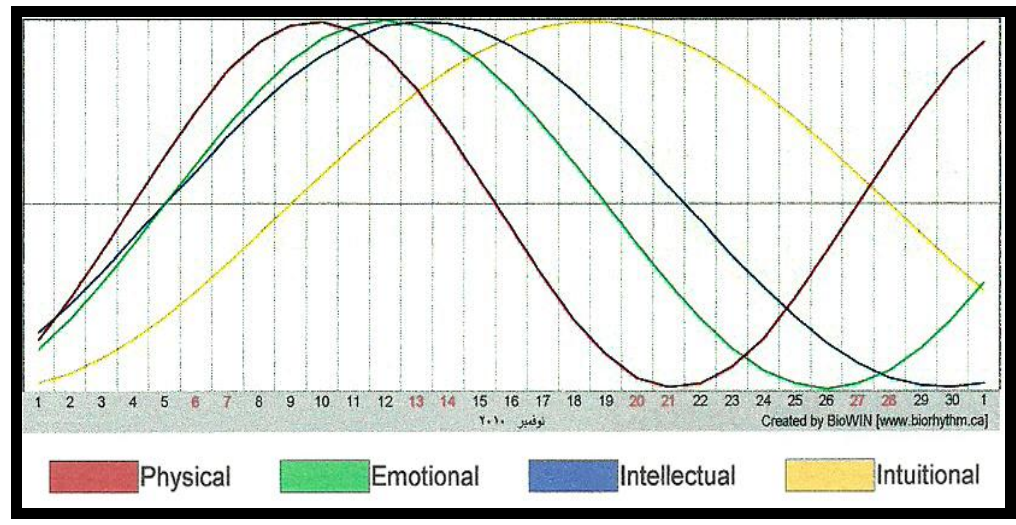


Figure (7)

Dynamic track for monthly bio- rhythm values for Kata players (No 5) during November 2010 under study

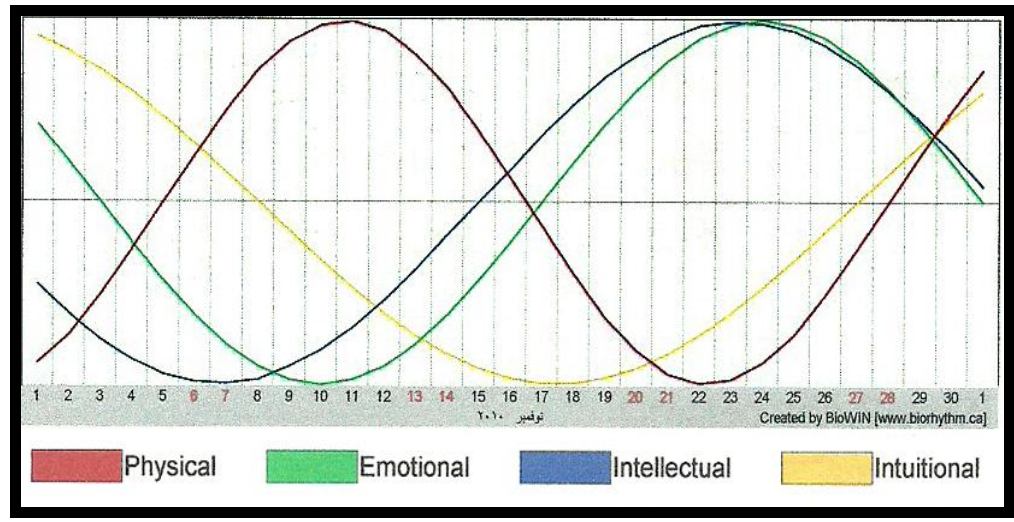

Figure (8)

Dynamic track for monthly bio- rhythm values for Kata players (No 6) during November 2010 under study

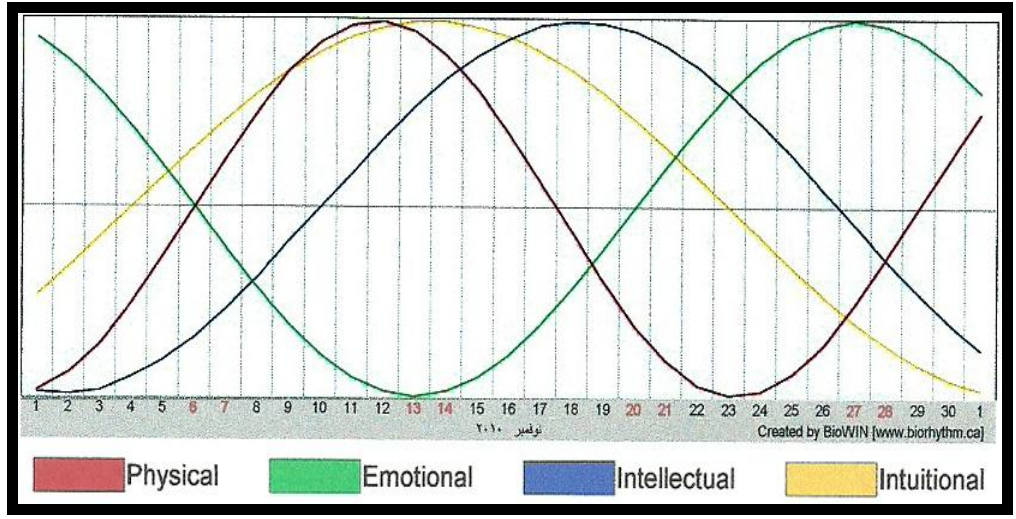

Figure (9)

Dynamic track for monthly bio- rhythm values for Kata players (No 7) during November 2010 under study

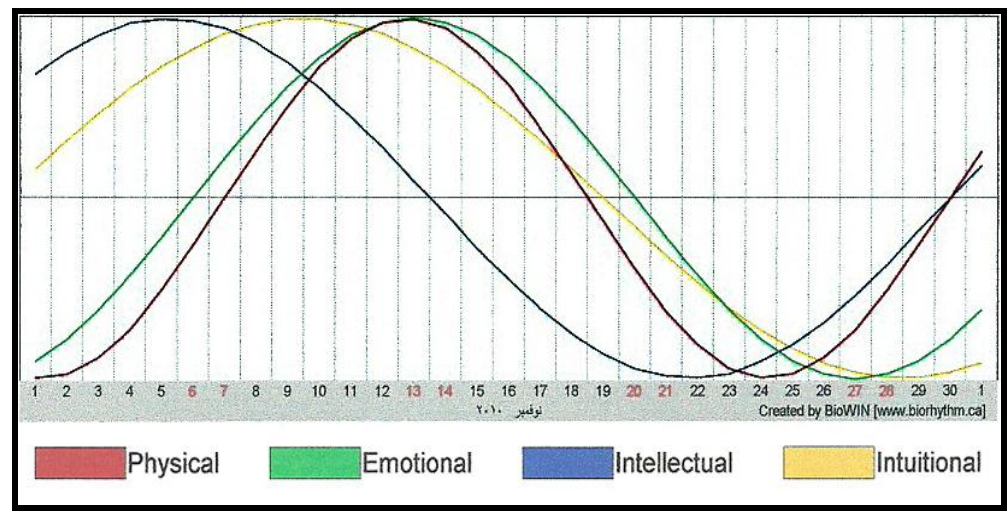


Figure (10)

Dynamic track for monthly bio- rhythm values for Kata players (No 8) during November 2010 under study

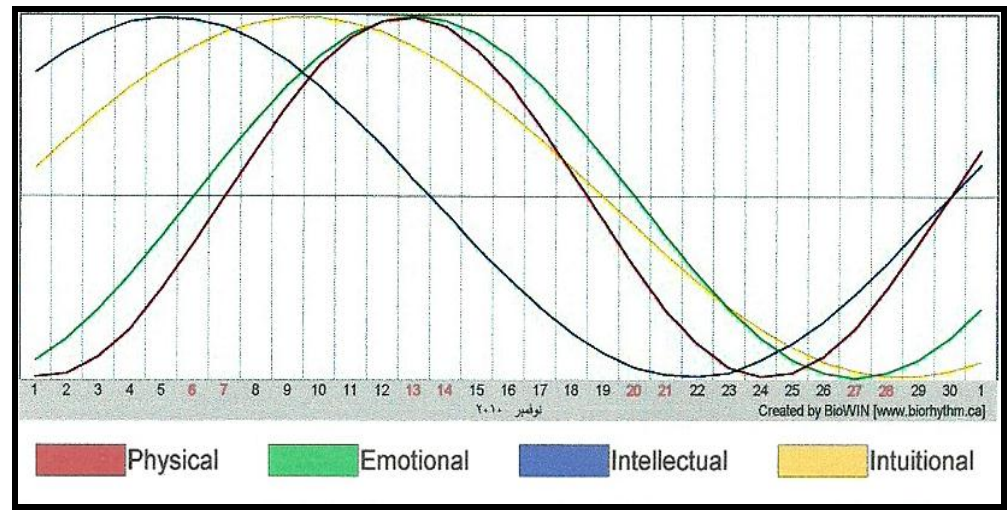

Figure (11)

Dynamic track for monthly bio- rhythm values for Kata players (No 9) during November 2010 under study

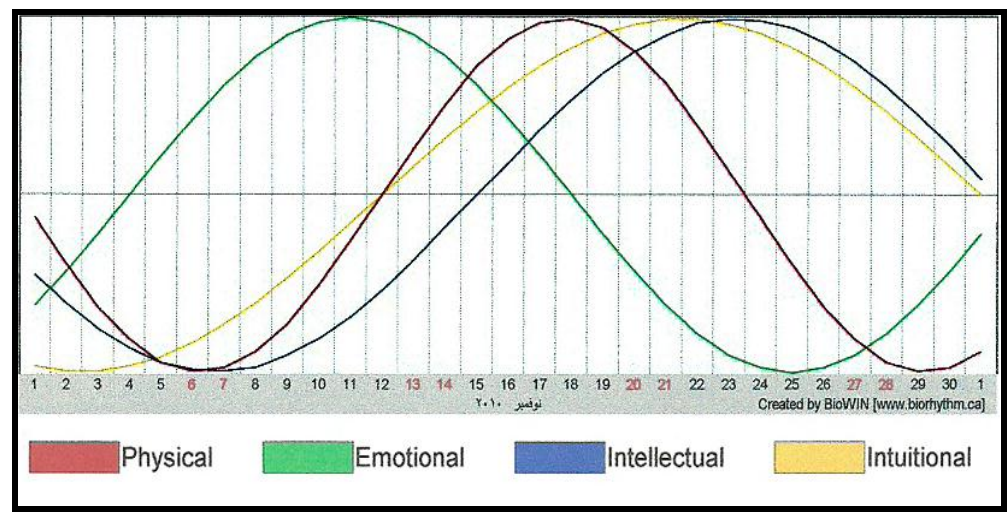

Figure (12)

Dynamic track for monthly bio- rhythm values for Kata players (No 10) during November 2010 under study

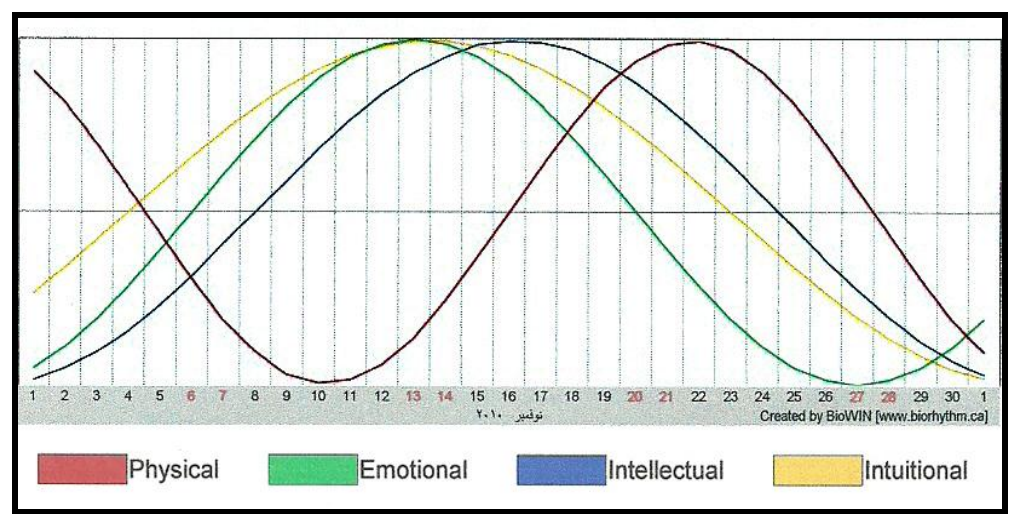


Table (1)

ANOVA test of "power" skill-physical variable values for skill styles of Unsu Kata component structure during various measurement times for Kata players

\begin{tabular}{|c|c|c|c|c|c|c|c|}
\hline \multicolumn{2}{|c|}{ Statistics } & Player's performing side & Variance source & $\begin{array}{l}\text { Sum of } \\
\text { Squares }\end{array}$ & DF & Mean Square & $\mathrm{F}$ \\
\hline & \multirow{3}{*}{$\begin{array}{l}\text { Lower block } \\
\text { (Gedan-Bari) }\end{array}$} & \multirow{3}{*}{$\begin{array}{l}\text { Left } \\
\text { Hidar }\end{array}$} & Between Groups & 124.909 & 6 & 20.818 & \multirow{3}{*}{$42.633^{*}$} \\
\hline & & & Within Groups & 340.182 & 70 & 0.488 & \\
\hline & & & Total & 159.091 & 76 & & \\
\hline \multirow{3}{*}{$\frac{0}{0}$} & \multirow{3}{*}{$\begin{array}{l}\text { Lower block } \\
\text { (Gedan-Bari) }\end{array}$} & \multirow{3}{*}{$\begin{array}{l}\text { Right } \\
\text { Migi }\end{array}$} & Between Groups & 111.974 & 6 & 18.662 & \multirow{3}{*}{$44.352 *$} \\
\hline & & & Within Groups & 29.455 & 70 & 0.421 & \\
\hline & & & Total & 141.429 & 76 & & \\
\hline \multirow{5}{*}{ 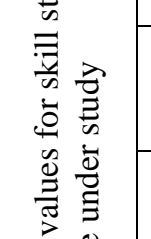 } & \multirow{3}{*}{$\begin{array}{c}\text { Knife hand } \\
\text { block } \\
\text { (Shuto-Uke) }\end{array}$} & \multirow{3}{*}{$\begin{array}{l}\text { Left } \\
\text { Hidar }\end{array}$} & Between Groups & 7.714 & 6 & 1.286 & \multirow{3}{*}{$3.094 *$} \\
\hline & & & Within Groups & 29.091 & 70 & 0.416 & \\
\hline & & & Total & 36.805 & 76 & & \\
\hline & \multirow{3}{*}{$\begin{array}{l}\text { Knife hand } \\
\text { block } \\
\text { (Shuto-Uke) }\end{array}$} & \multirow{3}{*}{$\begin{array}{l}\text { Right } \\
\text { Migi }\end{array}$} & Between Groups & 17.169 & 6 & 2.861 & \multirow{3}{*}{$10.015^{*}$} \\
\hline & & & Within Groups & 20.000 & 70 & 0.286 & \\
\hline \multirow{11}{*}{ 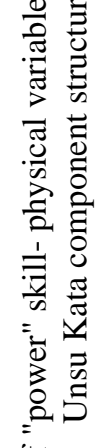 } & & & Total & 37.169 & 76 & & \\
\hline & \multirow{3}{*}{$\begin{array}{l}\text { Lunge punch } \\
\text { (Oi-Zuki) }\end{array}$} & \multirow{3}{*}{$\begin{array}{l}\text { Left } \\
\text { Hidar }\end{array}$} & Between Groups & 2.727 & 6 & 0.455 & \multirow{3}{*}{1.667} \\
\hline & & & Within Groups & 19.091 & 70 & 0.273 & \\
\hline & & & Total & 21.818 & 76 & & \\
\hline & \multirow{3}{*}{$\begin{array}{l}\text { Lunge punch } \\
\text { (Oi-Zuki) }\end{array}$} & \multirow{3}{*}{$\begin{array}{l}\text { Right } \\
\text { Migi }\end{array}$} & Between Groups & 3.091 & 6 & 0.515 & 1.983 \\
\hline & & & Within Groups & 18.182 & 70 & 0.260 & \\
\hline & & & Total & 21.273 & 76 & & \\
\hline & \multirow{3}{*}{$\begin{array}{c}\text { Reverse Punch } \\
\text { (Gyaku-Zuki) }\end{array}$} & \multirow{3}{*}{$\begin{array}{l}\text { Left } \\
\text { Hidar }\end{array}$} & Between Groups & 125.610 & 6 & 20.935 & $21.155^{*}$ \\
\hline & & & Within Groups & 69.273 & 70 & 0.990 & \\
\hline & & & Total & 194.883 & 76 & & \\
\hline & \multirow{3}{*}{$\begin{array}{c}\text { Reverse Punch } \\
\text { (Gyaku-Zuki) }\end{array}$} & \multirow{3}{*}{$\begin{array}{l}\text { Right } \\
\text { Migi }\end{array}$} & Between Groups & 81.273 & 6 & 13.545 & $20.135^{*}$ \\
\hline \multirow{8}{*}{ 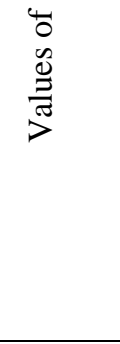 } & & & Within Groups & 47.091 & 70 & 0.673 & \\
\hline & & & Total & 128.364 & 76 & & \\
\hline & \multirow{3}{*}{$\begin{array}{c}\text { Side snap kick } \\
\text { (Yoku-Geri) }\end{array}$} & \multirow{3}{*}{$\begin{array}{l}\text { Left } \\
\text { Hidar }\end{array}$} & Between Groups & 30.805 & 6 & 5.134 & $11.696^{*}$ \\
\hline & & & Within Groups & 30.727 & 70 & 0.439 & \\
\hline & & & Total & 61.532 & 76 & & \\
\hline & \multirow{3}{*}{$\begin{array}{l}\text { Side snap kick } \\
\text { (Yoku-Geri) }\end{array}$} & & Between Groups & 33.091 & 6 & 5.515 & $16.589 *$ \\
\hline & & Kignt & Within Groups & 23.273 & 70 & 0.332 & \\
\hline & & & Total & 56.364 & 76 & & \\
\hline
\end{tabular}

Figure (13) Bio-rhythm daily track for values of "power" (Lower block left (Hidar Gedan-Bari)) skillphysical variable values for under study during various measurement times for Kata players

Figure (14) Bio-rhythm daily track for values of "power" (Lower block right (Migi Gedan-Bari)) skillphysical variable values for under study during various measurement times for Kata players
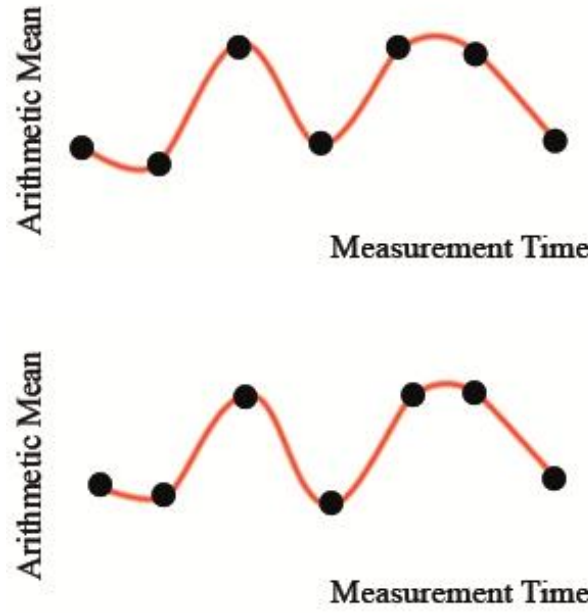
Figure (15) Bio-rhythm daily track for values of "power" (Knife hand block left (Hidar Shuto-Uke)) skill- physical variable values for under study during various measurement times for Kata players

Figure (16) Bio-rhythm daily track for values of "power" (Knife hand block right(Migi Shuto-Uke)) skillphysical variable values for under study during various measurement times for Kata players

Figure (17) Bio-rhythm daily track for values of "power" (Lunge punch left (Hidar Oi-Zuki)) skillphysical variable values for under study during various measurement times for Kata players

Figure (18) Bio-rhythm daily track for values of "power" (Lunge punch right (Migi Oi-Zuki)) skillphysical variable values for under study during various measurement times for Kata players

Figure (19) Bio-rhythm daily track for values of "power" (Reverse Punch left (Hidar Gyaku-Zuki)) skillphysical variable values for under study during various measurement times for Kata players

Figure (20) Bio-rhythm daily track for values of "power" (Side snap kick left (Hidar Yoku-Geri)) skillphysical variable values for under study during various measurement times for Kata players

Figure (21) Bio-rhythm daily track for values of "power" (Side snap kick right (Migi Yoku-Geri)) skillphysical variable values for under study during various measurement times for Kata players

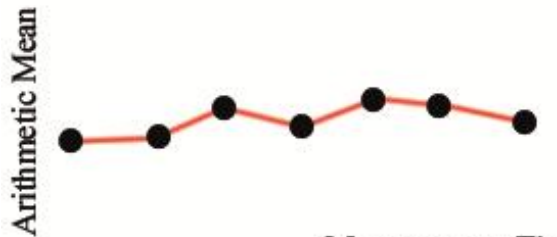

Measurement Time
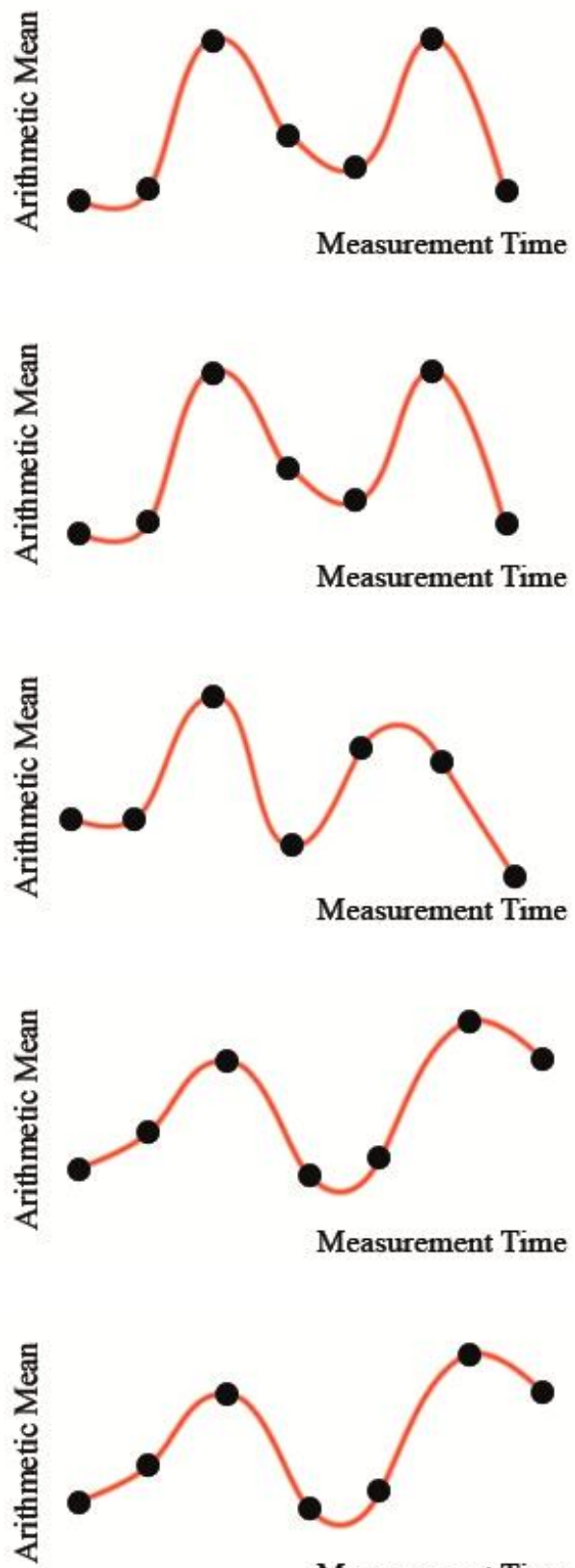

Measurement Time

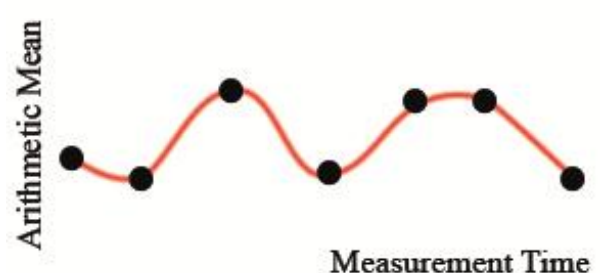

Measurement Time 
Table (2)

Differences significance for "power" skill-physical variable values for Lower block left (Hidar Gedan-Bari) using LSD test during various measurement times

\begin{tabular}{|c|c|c|c|c|c|c|c|c|}
\hline \multirow{2}{*}{ Measurement No } & \multirow{2}{*}{ Measurement time } & \multirow{2}{*}{ Mean } & \multicolumn{6}{|c|}{ Differences between means } \\
\cline { 5 - 9 } & & & $11 \mathrm{am}$ & $1 \mathrm{pm}$ & $3 \mathrm{pm}$ & $5 \mathrm{pm}$ & $7 \mathrm{pm}$ & $9 \mathrm{pm}$ \\
\hline 1 & $9 \mathrm{am}$ & 3.4545 & 0.3636 & $\uparrow 2.727^{*}$ & 0.1818 & $\uparrow 2.657^{*}$ & $\uparrow 2.455^{*}$ & 0.1818 \\
\hline 2 & $11 \mathrm{am}$ & 3.0909 & & $\uparrow 3.091^{*}$ & 0.5455 & $\uparrow 3.020^{*}$ & $\uparrow 2.818^{*}$ & 0.5455 \\
\hline 3 & $1 \mathrm{pm}$ & 6.1818 & & & $2.55^{*} \leftarrow$ & 0.071 & 0.2727 & $2.55^{*} \leftarrow$ \\
\hline 4 & $3 \mathrm{pm}$ & 3.6364 & & & & $\uparrow 2.475^{*}$ & $\uparrow 2.273^{*}$ & 0.0000 \\
\hline 5 & $5 \mathrm{pm}$ & 6.1111 & & & & 0.202 & $2.48^{*} \leftarrow$ \\
\hline 6 & $7 \mathrm{pm}$ & 5.9091 & & & & & $2.27^{*} \leftarrow$ \\
\hline
\end{tabular}

Table (3)

Differences significance for "power" skill-physical variable values for Lower block right (Migi Gedan-Bari) using LSD test during various measurement times

\begin{tabular}{|c|c|c|c|c|c|c|c|c|}
\hline \multirow{2}{*}{ Measurement No } & \multirow{2}{*}{ Measurement time } & \multirow{2}{*}{ Mean } & \multicolumn{7}{|c|}{ Differences between means } \\
\cline { 4 - 9 } & & & $11 \mathrm{am}$ & $1 \mathrm{pm}$ & $3 \mathrm{pm}$ & $5 \mathrm{pm}$ & $7 \mathrm{pm}$ & $9 \mathrm{pm}$ \\
\hline 1 & $9 \mathrm{am}$ & 3.1818 & 0.0909 & $\uparrow 2.455^{*}$ & 0.4545 & $\uparrow 2.374^{*}$ & $\uparrow 2.273^{*}$ & 0.2727 \\
\hline 2 & $11 \mathrm{am}$ & 3.0909 & & $\uparrow 2.546^{*}$ & 0.3636 & $\uparrow 2.465^{*}$ & $\uparrow 2.364^{*}$ & 0.3636 \\
\hline 3 & $1 \mathrm{pm}$ & 5.6364 & & & $2.91^{*} \leftarrow$ & 0.0808 & 0.1819 & $2.18^{*} \leftarrow$ \\
\hline 4 & $3 \mathrm{pm}$ & 2.7273 & & & & $\uparrow 2.828^{*}$ & $\uparrow 2.727^{*}$ & 0.727 \\
\hline 5 & $5 \mathrm{pm}$ & 5.5556 & & & & 0.1011 & $2.101^{*} \leftarrow$ \\
\hline 6 & $7 \mathrm{pm}$ & 5.4545 & & & & & & $2.00^{*} \leftarrow$ \\
\hline
\end{tabular}

Table (4)

Differences significance for "power" skill-physical variable values for Knife hand block left (Hidar Shuto-Uke) using LSD test during various measurement times

\begin{tabular}{|c|c|c|c|c|c|c|c|c|}
\hline \multirow{2}{*}{ Measurement No } & \multirow{2}{*}{ Measurement time } & \multirow{2}{*}{ Mean } & \multicolumn{6}{|c|}{ Differences between means } \\
\cline { 3 - 9 } & & & $11 \mathrm{am}$ & $1 \mathrm{pm}$ & $3 \mathrm{pm}$ & $5 \mathrm{pm}$ & $7 \mathrm{pm}$ & $9 \mathrm{pm}$ \\
\hline 1 & $9 \mathrm{am}$ & 4.3636 & 0.0909 & $\uparrow 0.727^{*}$ & 0.3637 & $\uparrow 0.969^{*}$ & $\uparrow 0.727^{*}$ & 0.4546 \\
\hline 2 & $11 \mathrm{am}$ & 4.4545 & & $\uparrow 0.636^{*}$ & 0.2728 & $\uparrow 0.879^{*}$ & $\uparrow 0.636^{*}$ & 0.3637 \\
\hline 3 & $1 \mathrm{pm}$ & 5.0909 & & & 0.3636 & 0.2424 & 0.0000 & 0.2727 \\
\hline 4 & $3 \mathrm{pm}$ & 4.7273 & & & & 0.606 & 0.3636 & 0.0909 \\
\hline 5 & $5 \mathrm{pm}$ & 5.3333 & & & & & 0.2424 & 0.5151 \\
\hline 6 & $7 \mathrm{pm}$ & 5.0909 & & & & & & 0.2727 \\
\hline
\end{tabular}

Table (5)

Differences significance for "power" skill-physical variable values for Knife hand block right (Migi Shuto-Uke) using LSD test during various measurement times

\begin{tabular}{|c|c|c|c|c|c|c|c|c|}
\hline \multirow{2}{*}{ Measurement No } & \multirow{2}{*}{ Measurement time } & \multirow{2}{*}{ Mean } & \multicolumn{7}{|c|}{ Differences between means } \\
\cline { 4 - 9 } & & & $11 \mathrm{am}$ & $1 \mathrm{pm}$ & $3 \mathrm{pm}$ & $5 \mathrm{pm}$ & $7 \mathrm{pm}$ & $9 \mathrm{pm}$ \\
\hline 1 & $9 \mathrm{am}$ & 4.3636 & 0.1819 & $\uparrow 1.000^{*}$ & 0.1819 & $\uparrow 1.303^{*}$ & $\uparrow 0.909^{*}$ & 0.1819 \\
\hline 2 & $11 \mathrm{am}$ & 4.5455 & & $\uparrow 0.818^{*}$ & 0.0000 & $\uparrow 1.121^{*}$ & $\uparrow 0.727^{*}$ & 0.0000 \\
\hline 3 & $1 \mathrm{pm}$ & 5.3636 & & & $0.81 * \rightarrow$ & 0.303 & 0.0909 & $0.81^{*} \rightarrow$ \\
\hline 4 & $3 \mathrm{pm}$ & 4.5455 & & & & $\uparrow 1.121^{*}$ & $\uparrow 0.727^{*}$ & 0.0000 \\
\hline 5 & $5 \mathrm{pm}$ & 5.6667 & & & & 0.394 & $1.12^{*} \leftarrow$ \\
\hline 6 & $7 \mathrm{pm}$ & 5.2727 & & & & & $0.73 * \leftarrow$ \\
\hline
\end{tabular}


Table (6)

Differences significance for "power" skill-physical variable values for Reverse Punch left (Hidar Gyaku-Zuki) using LSD test during various measurement times

\begin{tabular}{|c|c|c|c|c|c|c|c|c|}
\hline \multirow{2}{*}{ Measurement No } & \multirow{2}{*}{ Measurement time } & \multirow{2}{*}{ Mean } & \multicolumn{6}{|c|}{ Differences between means } \\
\hline & & & $11 \mathrm{am}$ & $1 \mathrm{pm}$ & $3 \mathrm{pm}$ & $5 \mathrm{pm}$ & $7 \mathrm{pm}$ & $9 \mathrm{pm}$ \\
\hline 1 & $9 \mathrm{am}$ & 3.0000 & 0.1818 & $\uparrow 2.909 *$ & 0.1818 & $\uparrow 3.000^{*}$ & $\uparrow 2.727 *$ & 0.5455 \\
\hline 2 & $11 \mathrm{am}$ & 3.1818 & & $\uparrow 2.727^{*}$ & 0.0000 & $\uparrow 2.818^{*}$ & $\uparrow 2.546^{*}$ & 0.3637 \\
\hline 3 & $1 \mathrm{pm}$ & 5.9091 & & & $\uparrow 2.727 *$ & 0.091 & 0.1818 & $2.36 * \leftarrow$ \\
\hline 4 & $3 \mathrm{pm}$ & 3.1818 & & & & $\uparrow 2.818^{*}$ & $\uparrow 2.546^{*}$ & $0.363 \leftarrow$ \\
\hline 5 & $5 \mathrm{pm}$ & 6.0000 & & & & & 0.2727 & $2.45 * \leftarrow$ \\
\hline 6 & $7 \mathrm{pm}$ & 5.7273 & & & & & & $2.18 * \leftarrow$ \\
\hline
\end{tabular}

Table (7)

Differences significance for "power" skill-physical variable values for Reverse Punch right (Migi Gyaku-Zuki) using LSD test during various measurement times

\begin{tabular}{|c|c|c|c|c|c|c|c|c|}
\hline \multirow{2}{*}{ Measurement No } & \multirow{2}{*}{ Measurement time } & \multirow{2}{*}{ Mean } & \multicolumn{6}{|c|}{ Differences between means } \\
\cline { 4 - 9 } & & & $11 \mathrm{am}$ & $1 \mathrm{pm}$ & $3 \mathrm{pm}$ & $5 \mathrm{pm}$ & $7 \mathrm{pm}$ & $9 \mathrm{pm}$ \\
\hline 1 & $9 \mathrm{am}$ & 3.1818 & 0.0909 & $\uparrow 2.273^{*}$ & 0.0000 & $\uparrow 2.152^{*}$ & $\uparrow 2.000^{*}$ & 0.0000 \\
\hline 2 & $11 \mathrm{am}$ & 3.2727 & & $\uparrow 2.182^{*}$ & 0.0909 & $\uparrow 2.061^{*}$ & $\uparrow 1.909^{*}$ & 0.0909 \\
\hline 3 & $1 \mathrm{pm}$ & 5.4545 & & & $2.27 * \rightarrow$ & 0.1212 & 0.2727 & $2.27 * \leftarrow$ \\
\hline 4 & $3 \mathrm{pm}$ & 3.1818 & & & & $\uparrow 2.152^{*}$ & $\uparrow 2.000^{*}$ & 0.0000 \\
\hline 5 & $5 \mathrm{pm}$ & 5.3333 & & & & & 0.1515 & $2.15^{*} \leftarrow$ \\
\hline 6 & $7 \mathrm{pm}$ & 5.1818 & & & & & & $2.00^{*} \leftarrow$ \\
\hline
\end{tabular}

Table (8)

Differences significance for "power" skill-physical variable values for Side snap kick left (Hidar Yoku-Geri) using LSD test during various measurement times

\begin{tabular}{|c|c|c|c|c|c|c|c|c|}
\hline \multirow{2}{*}{ Measurement No } & \multirow{2}{*}{ Measurement time } & \multirow{2}{*}{ Mean } & \multicolumn{6}{|c|}{ Differences between means } \\
\cline { 4 - 9 } & & & $11 \mathrm{am}$ & $1 \mathrm{pm}$ & $3 \mathrm{pm}$ & $5 \mathrm{pm}$ & $7 \mathrm{pm}$ & $9 \mathrm{pm}$ \\
\hline 1 & $9 \mathrm{am}$ & 3.3636 & 0.1819 & $\uparrow 1.364^{*}$ & 0.0909 & $\uparrow 1.081^{*}$ & $\uparrow 1.364^{*}$ & 0.1818 \\
\hline 2 & $11 \mathrm{am}$ & 3.5455 & & $\uparrow 1.182^{*}$ & 0.091 & $\uparrow 0.899^{*}$ & $\uparrow 1.182^{*}$ & 0.3637 \\
\hline 3 & $1 \mathrm{pm}$ & 4.7273 & & & $1.273^{*} \rightarrow$ & 0.283 & 0.0000 & $1.546^{*} \rightarrow$ \\
\hline 4 & $3 \mathrm{pm}$ & 3.4545 & & & & $\uparrow 0.989^{*}$ & $\uparrow 1.273^{*}$ & 0.2727 \\
\hline 5 & $5 \mathrm{pm}$ & 4.4444 & & & & 0.2829 & $1.263^{*}$ \\
\hline 6 & $7 \mathrm{pm}$ & 4.7273 & & & & & & $1.546^{*} \rightarrow$ \\
\hline
\end{tabular}

Table (9)

Differences significance for "power" skill-physical variable values for Side snap kick right (Migi Yoku-Geri) using LSD test during various measurement times

\begin{tabular}{|c|c|c|c|c|c|c|c|c|}
\hline \multirow{2}{*}{ Measurement No } & \multirow{2}{*}{ Measurement time } & \multirow{2}{*}{ Mean } & \multicolumn{6}{|c|}{ Differences between means } \\
\cline { 4 - 9 } & & & $11 \mathrm{am}$ & $1 \mathrm{pm}$ & $3 \mathrm{pm}$ & $5 \mathrm{pm}$ & $7 \mathrm{pm}$ & $9 \mathrm{pm}$ \\
\hline 1 & $9 \mathrm{am}$ & 3.5455 & 0.0909 & $\uparrow 1.364^{*}$ & 0.2728 & $\uparrow 0.899^{*}$ & $\uparrow 1.091^{*}$ & 0.3637 \\
\hline 2 & $11 \mathrm{am}$ & 3.4545 & & $\uparrow 1.455^{*}$ & 0.1818 & $\uparrow 0.989^{*}$ & $\uparrow 1.182^{*}$ & 0.2727 \\
\hline 3 & $1 \mathrm{pm}$ & 4.9091 & & & $1.636^{*} \rightarrow$ & $0.465^{*} \rightarrow$ & 0.2727 & $1.727^{*} \rightarrow$ \\
\hline 4 & $3 \mathrm{pm}$ & 3.2727 & & & & $\uparrow 1.172^{*}$ & $\uparrow 1.364^{*}$ & 0.0909 \\
\hline 5 & $5 \mathrm{pm}$ & 4.4444 & & & & 0.192 & $1.263^{* \rightarrow}$ \\
\hline 6 & $7 \mathrm{pm}$ & 4.6364 & & & & & $1.455^{*} \rightarrow$ \\
\hline
\end{tabular}

Table (1 to 9 ) results and figures (13-21) reveal morning, and between (5-7 pm) in evening.

that there was change in for "power" bio-rhythm dynamic track for Kata -under study- components structure, All variables ideal rhythm was between (11 am to $1 \mathrm{pm}$ ) in

This in consistent with what mentioned by Thomas Reilly (1990), Tamer Saleh Absi (2007), Doaa Sayed Ibrahim (2008). 
Table (10)

ANOVA test of "special performance endurance" skill-physical variable values for skill styles of Unsu Kata component structure during various measurement times for Kata players

\begin{tabular}{|c|c|c|c|c|c|c|c|}
\hline \multicolumn{2}{|c|}{ Study variables } & $\begin{array}{l}\text { Player's performing } \\
\text { side }\end{array}$ & Variance source & $\begin{array}{l}\text { Sum of } \\
\text { Squares }\end{array}$ & DF & $\begin{array}{l}\text { Mean } \\
\text { Square }\end{array}$ & $\mathrm{F}$ \\
\hline & \multirow{3}{*}{$\begin{array}{l}\text { Lower block } \\
\text { (Gedan-Bari) }\end{array}$} & \multirow{3}{*}{$\begin{array}{l}\text { Left } \\
\text { Hidar }\end{array}$} & Between Groups & 1667.403 & 6 & 277.900 & \multirow{3}{*}{$390.481 *$} \\
\hline & & & Within Groups & 49.818 & 70 & 0.712 & \\
\hline & & & Total & 1717.221 & 76 & & \\
\hline & \multirow{3}{*}{$\begin{array}{l}\text { Lower block } \\
\text { (Gedan-Bari) }\end{array}$} & \multirow{3}{*}{$\begin{array}{l}\text { Right } \\
\text { Migi }\end{array}$} & Between Groups & 1290.519 & 6 & 215.087 & \multirow{3}{*}{$250.176^{*}$} \\
\hline & & & Within Groups & 60.182 & 70 & 0.860 & \\
\hline \multirow{6}{*}{ 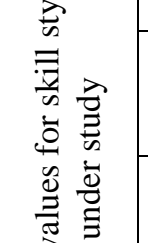 } & & & Total & 1350.701 & 76 & & \\
\hline & \multirow{3}{*}{$\begin{array}{l}\text { Knife hand } \\
\text { block } \\
\text { (Shuto-Uke) }\end{array}$} & \multirow{3}{*}{$\begin{array}{l}\text { Left } \\
\text { Hidar }\end{array}$} & Between Groups & 290.701 & 6 & 48.450 & \multirow{3}{*}{$121.126^{*}$} \\
\hline & & & Within Groups & 28.000 & 70 & 0.400 & \\
\hline & & & Total & 318.701 & 76 & & \\
\hline & \multirow{3}{*}{$\begin{array}{l}\text { Knife hand } \\
\text { block } \\
\text { (Shuto-Uke) }\end{array}$} & \multirow{3}{*}{$\begin{array}{l}\text { Right } \\
\text { Migi }\end{array}$} & Between Groups & 295.039 & 6 & 49.173 & \multirow{3}{*}{$88.466^{*}$} \\
\hline & & & Within Groups & 38.909 & 70 & 0.556 & \\
\hline \multirow{11}{*}{ 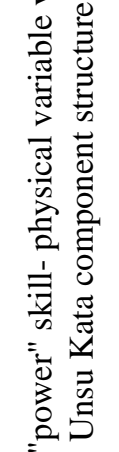 } & & & Total & 333.948 & 76 & & \\
\hline & \multirow{3}{*}{$\begin{array}{l}\text { Lunge punch } \\
\text { (Oi-Zuki) }\end{array}$} & \multirow{3}{*}{$\begin{array}{l}\text { Left } \\
\text { Hidar }\end{array}$} & Between Groups & 649.896 & 6 & 108.316 & \multirow{3}{*}{$93.292 *$} \\
\hline & & & Within Groups & 81.273 & 70 & 1.161 & \\
\hline & & & Total & 731.169 & 76 & & \\
\hline & \multirow{3}{*}{$\begin{array}{l}\text { Lunge punch } \\
\text { (Oi-Zuki) }\end{array}$} & \multirow{3}{*}{$\begin{array}{l}\text { Right } \\
\text { Migi }\end{array}$} & Between Groups & 758.883 & 6 & 126.481 & \multirow{3}{*}{$146.231^{*}$} \\
\hline & & & Within Groups & 60.545 & 70 & 0.865 & \\
\hline & & & Total & 819.429 & 76 & & \\
\hline & \multirow{3}{*}{$\begin{array}{l}\text { Reverse Punch } \\
\text { (Gyaku-Zuki) }\end{array}$} & \multirow{3}{*}{$\begin{array}{l}\text { Left } \\
\text { Hidar }\end{array}$} & Between Groups & 915.974 & 6 & 152.662 & \multirow{3}{*}{$309.342 *$} \\
\hline & & & Within Groups & 34.545 & 70 & 0.494 & \\
\hline & & & Total & 950.519 & 76 & & \\
\hline & \multirow{3}{*}{$\begin{array}{l}\text { Reverse Punch } \\
\text { (Gyaku-Zuki) }\end{array}$} & \multirow{3}{*}{$\begin{array}{l}\text { Right } \\
\text { Migi }\end{array}$} & Between Groups & 621.714 & 6 & 103.619 & \multirow{3}{*}{$211.076^{*}$} \\
\hline \multirow{8}{*}{ 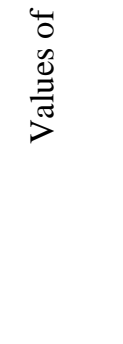 } & & & Within Groups & 34.364 & 70 & 0.491 & \\
\hline & & & Total & 656.078 & 76 & & \\
\hline & Side snap & & Between Groups & 272.857 & 6 & 45.476 & \\
\hline & kick & $\begin{array}{l}\text { Lett } \\
\text { Hidar }\end{array}$ & Within Groups & 21.273 & 70 & 0.304 & 149.644* \\
\hline & (Yoku-Geri) & & Total & 294.130 & 76 & & \\
\hline & & & Between Groups & 203.714 & 6 & 33.952 & \\
\hline & kick & Right & Within Groups & 17.818 & 70 & 0.255 & 133.384* \\
\hline & (Yoku-Geri) & & Total & 221.532 & 76 & & \\
\hline
\end{tabular}

Figure (22) Bio-rhythm daily track for values of "special performance endurance" (Lower block left (Hidar Gedan-Bari)) skill- physical variable values for under study during various measurement times for Kata players

Figure (23) Bio-rhythm daily track for values of "special performance endurance" (Lower block right (Migi Gedan-Bari)) skill- physical variable values for under study during various measurement times for Kata players
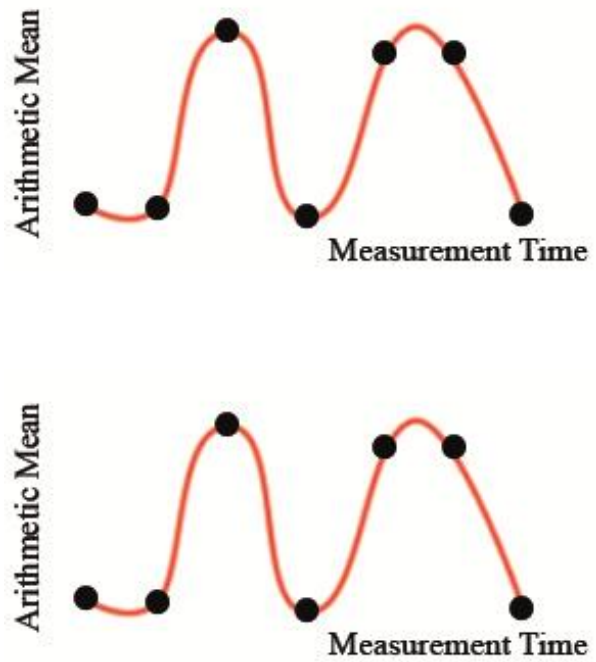
Figure (24) Bio-rhythm daily track for values of "special performance endurance" (Knife hand block left (Hidar Shuto-Uke)) skill- physical variable values for under study during various measurement times for Kata players

Figure (25) Bio-rhythm daily track for values of "special performance endurance" (Knife hand block right(Migi Shuto-Uke)) skill- physical variable values for under study during various measurement times for Kata players

Figure (26) Bio-rhythm daily track for values of "special performance endurance" (Lunge punch left (Hidar OiZuki)) skill- physical variable values for under study during various measurement times for Kata players

Figure (27) Bio-rhythm daily track for values of "special performance endurance" (Lunge punch right (Migi OiZuki)) skill- physical variable values for under study during various measurement times for Kata players

Figure (28) Bio-rhythm daily track for values of "special performance endurance" (Reverse Punch left (Hidar Gyaku-Zuki)) skill- physical variable values for under study during various measurement times for Kata players

Figure (29) Bio-rhythm daily track for values "special performance endurance" (Reverse Punch right (Migi Gyaku-Zuki)) skill- physical variable values for under study during various measurement times for Kata players
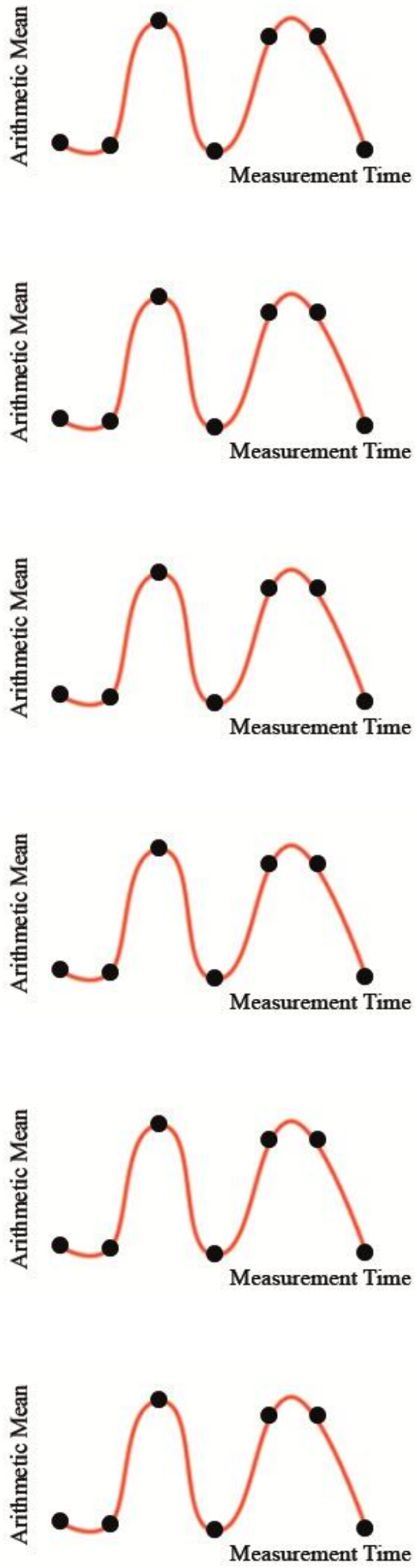
Figure (30) Bio-rhythm daily track for values of "special performance endurance" (Side snap kick left (Hidar Yoku-Geri)) skill- physical variable values for under study during various measurement times for Kata players

Figure (31) Bio-rhythm daily track for values of "special performance endurance" (Side snap kick right (Migi Yoku-Geri)) skill- physical variable values for under study during various measurement times for Kata players
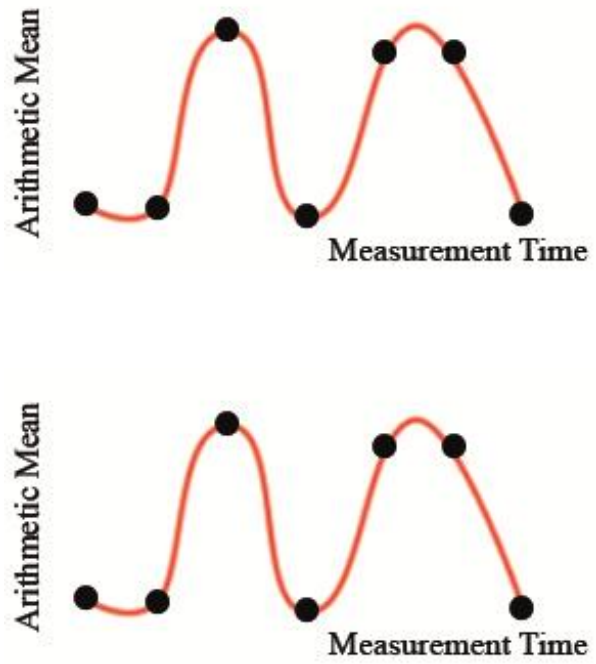

Table (11)

Differences significance for "power" skill-physical variable values for Lower block left (Hidar Gedan-Bari) using LSD test during vxarious measurement times

\begin{tabular}{|c|c|c|c|c|c|c|c|c|}
\hline \multirow{2}{*}{ Measurement No } & \multirow{2}{*}{ Measurement time } & \multirow{2}{*}{ Mean } & \multicolumn{5}{|c|}{ Differences between means } \\
\cline { 4 - 9 } & & & $11 \mathrm{am}$ & $1 \mathrm{pm}$ & $3 \mathrm{pm}$ & $5 \mathrm{pm}$ & $7 \mathrm{pm}$ & $9 \mathrm{pm}$ \\
\hline 1 & $9 \mathrm{am}$ & 4.0000 & 0.5455 & $\uparrow 9.364^{*}$ & $\uparrow 0.818^{*}$ & $\uparrow 8.778^{*}$ & $\uparrow 8.455^{*}$ & 0.6364 \\
\hline 2 & $11 \mathrm{am}$ & 3.4545 & & $\uparrow 9.909^{*}$ & 0.2727 & $\uparrow 9.323^{*}$ & $\uparrow 9.000^{*}$ & 0.0909 \\
\hline 3 & $1 \mathrm{pm}$ & 13.3636 & & & $10.18^{*}$ & 0.5858 & $\uparrow 0.909^{*}$ & $10.00^{*} \leftarrow$ \\
\hline 4 & $3 \mathrm{pm}$ & 3.1818 & & & & $\uparrow 9.596^{*}$ & $\uparrow 9.273^{*}$ & 0.1818 \\
\hline 5 & $5 \mathrm{pm}$ & 12.7778 & & & & & 0.3232 & $9.41^{*} \leftarrow$ \\
\hline 6 & $7 \mathrm{pm}$ & 12.4545 & & & & & & \\
\hline
\end{tabular}

Table (12)

Differences significance for "power" skill- physical variable values for Lower block right (Migi Gedan-Bari) using LSD test during various measurement times

\begin{tabular}{|c|c|c|c|c|c|c|c|c|}
\hline \multirow{2}{*}{$\begin{array}{c}\text { Measurement } \\
\text { No }\end{array}$} & \multirow{2}{*}{$\begin{array}{l}\text { Measurement } \\
\text { time }\end{array}$} & \multirow{2}{*}{ Mean } & \multicolumn{6}{|c|}{ Differences between means } \\
\hline & & & $11 \mathrm{am}$ & $1 \mathrm{pm}$ & $3 \mathrm{pm}$ & $5 \mathrm{pm}$ & $7 \mathrm{pm}$ & $9 \mathrm{pm}$ \\
\hline 1 & 9 am & 3.1818 & 0.4545 & $\uparrow 8.546 *$ & 0.09091 & $\uparrow 8.262 *$ & $\uparrow 7.818^{*}$ & 0.0000 \\
\hline 2 & $11 \mathrm{am}$ & 2.7273 & & $\uparrow 9.000 *$ & 0.3636 & $\uparrow 8.717 *$ & $\uparrow 8.273 *$ & 0.4545 \\
\hline 3 & $1 \mathrm{pm}$ & 11.7273 & & & $\stackrel{\leftarrow}{8.636 *}$ & 0.2829 & 0.7273 & $8.546^{*}$ \\
\hline 4 & $3 \mathrm{pm}$ & 3.0909 & & & & $\uparrow 8.354 *$ & $\uparrow 7.909 *$ & 0.0909 \\
\hline 5 & $5 \mathrm{pm}$ & 11.4444 & & & & & 0.4444 & $8.263 *$ \\
\hline 6 & $7 \mathrm{pm}$ & 11.0000 & & & & & & $\leftarrow . \leftarrow 18^{*}$ \\
\hline
\end{tabular}


Table (13)

Differences significance for "power" skill-physical variable values for Knife hand block left (Hidar Shuto-Uke) using LSD test during various measurement times

\begin{tabular}{|c|c|c|c|c|c|c|c|c|}
\hline \multirow{2}{*}{ Measurement No } & \multirow{2}{*}{ Measurement time } & \multirow{2}{*}{ Mean } & \multicolumn{6}{|c|}{ Differences between means } \\
\hline & & & $11 \mathrm{am}$ & $1 \mathrm{pm}$ & $3 \mathrm{pm}$ & $5 \mathrm{pm}$ & $7 \mathrm{pm}$ & $9 \mathrm{pm}$ \\
\hline 1 & $9 \mathrm{am}$ & 4. 1818 & 0.2727 & $\uparrow 4.364 *$ & 0.4546 & $\uparrow 4.263^{*}$ & $\uparrow 3.909 *$ & 0.3637 \\
\hline 2 & $11 \mathrm{am}$ & 4.4545 & & $\uparrow 4.091^{*}$ & 0.1819 & $\uparrow 3.989^{*}$ & $\uparrow 3.636^{*}$ & 0.0909 \\
\hline 3 & $1 \mathrm{pm}$ & 8.5455 & & & $\underset{3.909^{*}}{\leftarrow}$ & 0.1011 & 0.4546 & $\leftarrow$ \\
\hline 4 & $3 \mathrm{pm}$ & 4.6364 & & & & $\uparrow 3.808 *$ & $\uparrow 3.455^{*}$ & 0.0909 \\
\hline 5 & $5 \mathrm{pm}$ & 8.4444 & & & & & 0.3535 & $\underset{3.899}{\leftarrow}$ \\
\hline 6 & $7 \mathrm{pm}$ & 8.0909 & & & & & & $\underset{3.546 *}{\leftarrow}$ \\
\hline
\end{tabular}

Table (14)

Differences significance for "power" skill-physical variable values for Knife hand block right (Migi Shuto-Uke) using LSD test during various measurement times

\begin{tabular}{|c|c|c|c|c|c|c|c|c|}
\hline \multirow{2}{*}{ Measurement No } & \multirow{2}{*}{ Measurement time } & \multirow{2}{*}{ Mean } & \multicolumn{5}{|c|}{ Differences between means } \\
\cline { 3 - 8 } & & & $11 \mathrm{am}$ & $1 \mathrm{pm}$ & $3 \mathrm{pm}$ & $5 \mathrm{pm}$ & $7 \mathrm{pm}$ & $9 \mathrm{pm}$ \\
\hline 1 & $9 \mathrm{am}$ & 4.2727 & 0.3637 & $\uparrow 4.455^{*}$ & 0.2727 & $\uparrow 4.172^{*}$ & $\uparrow 3.818^{*}$ & 0.0000 \\
\hline 2 & $11 \mathrm{am}$ & 4.6364 & & $\uparrow 4.091^{*}$ & 0.0909 & $\uparrow 3.808^{*}$ & $\uparrow 3.455^{*}$ & 0.3637 \\
\hline 3 & $1 \mathrm{pm}$ & 8.7273 & & & $4.18^{*} \leftarrow$ & 0.2829 & 0.636 & $4.45^{*} \leftarrow$ \\
\hline 4 & $3 \mathrm{pm}$ & 4.5455 & & & $\uparrow 3.899^{*}$ & $\uparrow 3.545^{*}$ & 0.2728 \\
\hline 5 & $5 \mathrm{pm}$ & 8.4444 & & & & 0.3535 & $\leftarrow 4.17^{*}$ \\
\hline 6 & $7 \mathrm{pm}$ & 8.0909 & & & & & \\
\hline
\end{tabular}

Table (15)

Differences significance for "power" skill-physical variable values for Lunge punch left (Hidar Oi-Zuki) using LSD test during various measurement times

\begin{tabular}{|c|c|c|c|c|c|c|c|c|}
\hline \multirow{2}{*}{$\begin{array}{c}\text { Measurement } \\
\text { No }\end{array}$} & \multirow{2}{*}{$\begin{array}{l}\text { Measurement } \\
\text { time }\end{array}$} & \multirow{2}{*}{ Mean } & \multicolumn{6}{|c|}{ Differences between means } \\
\hline & & & $11 \mathrm{am}$ & $1 \mathrm{pm}$ & $3 \mathrm{pm}$ & $5 \mathrm{pm}$ & $7 \mathrm{pm}$ & $9 \mathrm{pm}$ \\
\hline 1 & $9 \mathrm{am}$ & 5.7273 & 0.1818 & $\uparrow 6.000^{*}$ & 0.1818 & $\uparrow 5.717 *$ & $\uparrow 5.545 *$ & 0.1818 \\
\hline 2 & $11 \mathrm{am}$ & 5.5455 & & $\uparrow 6.182 *$ & 0.0000 & $\uparrow 5.899 *$ & $\uparrow 5.727 *$ & 0.0000 \\
\hline 3 & $1 \mathrm{pm}$ & 11.7273 & & & $6 . \overleftarrow{182 *}$ & 0.2829 & 0.4546 & $\leftarrow \overleftarrow{6.182} *$ \\
\hline 4 & $3 \mathrm{pm}$ & 5.5455 & & & & $\uparrow 5.899 *$ & $\uparrow 5.727 *$ & 0.0000 \\
\hline 5 & $5 \mathrm{pm}$ & 11.4444 & & & & & 0.1717 & $\leftarrow \overleftarrow{5.899} *$ \\
\hline 6 & $7 \mathrm{pm}$ & 11.2727 & & & & & & $\leftarrow$ \\
\hline
\end{tabular}


Table (16)

Differences significance for "power" skill-physical variable values for Lunge punch right (Migi Oi-Zuki) using LSD test during various measurement times

\begin{tabular}{|c|c|c|c|c|c|c|c|c|}
\hline \multirow{2}{*}{$\begin{array}{c}\text { Measurement } \\
\text { No }\end{array}$} & \multirow{2}{*}{$\begin{array}{c}\text { Measurement } \\
\text { time }\end{array}$} & \multirow{2}{*}{ Mean } & \multicolumn{6}{|c|}{ Differences between means } \\
\hline & & & $11 \mathrm{am}$ & $1 \mathrm{pm}$ & $3 \mathrm{pm}$ & $5 \mathrm{pm}$ & $7 \mathrm{pm}$ & $9 \mathrm{pm}$ \\
\hline 1 & $9 \mathrm{am}$ & 5.3636 & 0.1818 & $\uparrow 6.818^{*}$ & 0.0909 & $\uparrow 6.303^{*}$ & $\uparrow 6.000^{*}$ & 0.1819 \\
\hline 2 & $11 \mathrm{am}$ & 5.5455 & & $\uparrow 6.636^{*}$ & 0.2727 & $\uparrow 6.121 *$ & $\uparrow 5.818 *$ & 0.0000 \\
\hline 3 & $1 \mathrm{pm}$ & 12.1818 & & & $6.91 * \leftarrow$ & 0.515 & $\uparrow 0.818 *$ & $\leftarrow \leftarrow$ \\
\hline 4 & $3 \mathrm{pm}$ & 5.2727 & & & & $\uparrow 6.394 *$ & $\uparrow 6.091 *$ & 0.2728 \\
\hline 5 & $5 \mathrm{pm}$ & 11.6667 & & & & & 0.3031 & $\leftarrow 6.12 *$ \\
\hline 6 & $7 \mathrm{pm}$ & 11.3636 & & & & & & $\leftarrow \leftarrow$ \\
\hline
\end{tabular}

Table (17)

Differences significance for "power" skill-physical variable values for Reverse Punch left (Hidar Gyaku-Zuki) using LSD test during various measurement times

\begin{tabular}{|c|c|c|c|c|c|c|c|c|}
\hline \multirow{2}{*}{ Measurement No } & \multirow{2}{*}{ Measurement time } & \multirow{2}{*}{ Mean } & \multicolumn{6}{|c|}{ Differences between means } \\
\hline & & & $11 \mathrm{am}$ & $1 \mathrm{pm}$ & $3 \mathrm{pm}$ & $5 \mathrm{pm}$ & $7 \mathrm{pm}$ & $9 \mathrm{pm}$ \\
\hline 1 & $9 \mathrm{am}$ & 2.9091 & 0.4546 & $\uparrow 6.818^{*}$ & 0.2727 & $\uparrow 6.758 *$ & $\uparrow 6.545^{*}$ & 0.4546 \\
\hline 2 & $11 \mathrm{am}$ & 2.4545 & & $\uparrow 7.273^{*}$ & 0.1819 & $\uparrow 7.212^{*}$ & $\uparrow 7.000^{*}$ & 0.0000 \\
\hline 3 & $1 \mathrm{pm}$ & 9.7273 & & & $\underset{7.091}{\leftarrow}$ & 0.0606 & 0.2728 & $\underset{7.273}{\leftarrow}$ \\
\hline 4 & $3 \mathrm{pm}$ & 2.6364 & & & & $\uparrow 7.030 *$ & $\uparrow 6.818 *$ & 0.1819 \\
\hline 5 & $5 \mathrm{pm}$ & 9.6667 & & & & & 0.2122 & $\underset{7.212 *}{\leftarrow}$ \\
\hline 6 & $7 \mathrm{pm}$ & 9.4545 & & & & & & $7.00 * \leftarrow$ \\
\hline
\end{tabular}

Table (18)

Differences significance for "power" skill-physical variable values for Reverse Punch right (Migi Gyaku-Zuki) using LSD test during various measurement times

\begin{tabular}{|c|c|c|c|c|c|c|c|c|}
\hline \multirow{2}{*}{ Measurement No } & \multirow{2}{*}{ Measurement time } & \multirow{2}{*}{ Mean } & \multicolumn{6}{|c|}{ Differences between means } \\
\hline & & & $11 \mathrm{am}$ & $1 \mathrm{pm}$ & $3 \mathrm{pm}$ & $5 \mathrm{pm}$ & $7 \mathrm{pm}$ & $9 \mathrm{pm}$ \\
\hline 1 & $9 \mathrm{am}$ & 2.8182 & 0.4546 & $\uparrow 6.091 *$ & 0.5454 & $\uparrow 5.959 *$ & $\uparrow 5.545^{*}$ & 0.3636 \\
\hline 2 & $11 \mathrm{am}$ & 2.3636 & & $\uparrow 6.546^{*}$ & $\uparrow 1.000 *$ & $\uparrow 6.414 *$ & $\uparrow 6.000^{*}$ & $\uparrow 0.818^{*}$ \\
\hline 3 & $1 \mathrm{pm}$ & 8.9091 & & & $\overleftarrow{5.546^{*}}$ & 0.1313 & 0.5455 & $5.727^{*}$ \\
\hline 4 & $3 \mathrm{pm}$ & 3.3636 & & & & $\uparrow 5.414^{*}$ & $\uparrow 5.000^{*}$ & 0.1818 \\
\hline 5 & $5 \mathrm{pm}$ & 8.7778 & & & & & 0.4142 & $5.596 *$ \\
\hline 6 & $7 \mathrm{pm}$ & 8.3636 & & & & & & $5.182 *$ \\
\hline
\end{tabular}

Table (19)

Differences significance for "power" skill-physical variable values for Side snap kick left (Hidar Yoku-Geri) using LSD test during various measurement times

\begin{tabular}{|c|c|c|c|c|c|c|c|c|}
\hline \multirow{2}{*}{ Measurement No } & \multirow{2}{*}{ Measurement time } & \multirow{2}{*}{ Mean } & \multicolumn{6}{|c|}{ Differences between means } \\
\hline & & & $11 \mathrm{am}$ & $1 \mathrm{pm}$ & $3 \mathrm{pm}$ & $5 \mathrm{pm}$ & $7 \mathrm{pm}$ & $9 \mathrm{pm}$ \\
\hline 1 & $9 \mathrm{am}$ & 3.3636 & 0.0000 & $\uparrow 3.818^{*}$ & 0.0909 & $\uparrow 3.303^{*}$ & $\uparrow 3.636^{*}$ & $\uparrow 0.636^{*}$ \\
\hline 2 & $11 \mathrm{am}$ & 3.3636 & & $\uparrow 3.818 *$ & 0.0909 & $\uparrow 3.303^{*}$ & $\uparrow 3.636^{*}$ & $\uparrow 0.636^{*}$ \\
\hline 3 & $1 \mathrm{pm}$ & 7.1818 & & & $3.73 * \leftarrow$ & 0.510 & 0.1818 & $4.46^{*} \leftarrow$ \\
\hline 4 & $3 \mathrm{pm}$ & 3.4545 & & & & $\uparrow 3.212 *$ & $\uparrow 3.546^{*}$ & $0.72 * \leftarrow$ \\
\hline 5 & $5 \mathrm{pm}$ & 6.6667 & & & & & 0.3333 & $\underset{3.939 *}{\leftarrow}$ \\
\hline 6 & $7 \mathrm{pm}$ & 7.0000 & & & & & & $\stackrel{\leftarrow}{4.273 *}$ \\
\hline
\end{tabular}


Table (20)

Differences significance for "power" skill-physical variable values for Side snap kick right (Migi Yoku-Geri) using LSD test during various measurement times

\begin{tabular}{|c|c|c|c|c|c|c|c|c|}
\hline \multirow{2}{*}{ Measurement No } & \multirow{2}{*}{ Measurement time } & \multirow{2}{*}{ Mean } & \multicolumn{6}{|c|}{ Differences between means } \\
\hline & & & $11 \mathrm{am}$ & $1 \mathrm{pm}$ & $3 \mathrm{pm}$ & $5 \mathrm{pm}$ & $7 \mathrm{pm}$ & $9 \mathrm{pm}$ \\
\hline 1 & $9 \mathrm{am}$ & 3.5455 & 0.1819 & $\uparrow 3.091^{*}$ & 0.3637 & $\uparrow 2.899^{*}$ & $\uparrow 2.909 *$ & $\uparrow 0.636^{*}$ \\
\hline 2 & $11 \mathrm{am}$ & 3.3636 & & $\uparrow 3.273^{*}$ & 0.1818 & $\uparrow 3.081^{*}$ & $\uparrow 3.091^{*}$ & $\uparrow 0.455^{*}$ \\
\hline 3 & $1 \mathrm{pm}$ & 6.6364 & & & $3.46 * \leftarrow$ & 0.192 & 0.1819 & $3.73 * \rightarrow$ \\
\hline 4 & $3 \mathrm{pm}$ & 3.1818 & & & & $\uparrow 3.263^{*}$ & $\uparrow 3.273^{*}$ & 0.2727 \\
\hline 5 & $5 \mathrm{pm}$ & 6.4444 & & & & & 0.010 & $3.54 * \leftarrow$ \\
\hline 6 & $7 \mathrm{pm}$ & 6.4545 & & & & & & $3.55 * \leftarrow$ \\
\hline
\end{tabular}

Tables (10 to 20) results and figures (22 to 31 ) reveal that there was change in "special performance endurance " bio-rhythm dynamic track for Kata -under study- components structure, All variables ideal rhythm was between (11 am to $1 \mathrm{pm})$ in morning, and between $(5-7 \mathrm{pm})$ in evening.

These may be due to high temperature which was reflected metabolism rate and enzymes contributing to body system endurance for physical effort for skill styles Kata (under study) component structure . this is consistent with what mentioned by Reilly. T. (1990), Aly Elbeck and Sabry Omar (1994), Ahmed Mahmoud Ibrahim (2005).

\section{Conclusions:}

Bio-rhythm dynamic track for "Power and special performance endurance" for Kata players reach its highest rate during morning from 11 am to $1 \mathrm{pm}$, during evening from $5 \mathrm{pm}$ to $7 \mathrm{pm}$.

\section{Recommendations}

1. As per the what concluded above from study's statistics results and in accordance with study procedures the following could be recommended:

2. Using the highest daily bio-rhythm for physical-skill parameters as indicator when planning training times for Kaa players

3. Conducting further surveys and experimental studies bio-rhythm dynamic track ideal times for other physical-skill parameters for Kata in different Karate schools to be used as an indicator for planning times

\section{References:}

1. Abul Ela Ahmed Abdel Fattah (1997): athletic training, physiological foundations, 1st ed. Dar ElFikr ElArabi , Cairo.

2. Ahmed Adel El-Shechany(1992).: the impact of local time difference on athletic achievement, theories and applications magazine, No.13, Faculty of Physical Education for Men, Abu Qir, Alexandria

3. Ahmed Ali Ibrahim Al-Attar (2000): an analytical study of bio-rhythm dynamics and its relationp to sports injuries for players in some levels in some fighting sports, master thesis , Faculty of Physical Education, University of Tanta.

4. Ahmed Mahmoud Mohamed Ibrahim (1992): the relationship between the functional efficiency of central nervous system and some attention features as a selection parameter for karate players, Journal of Studies and Research of Physical Education, Faculty of Physical Education, Helwan University.

5. Ahmed Mahmoud Mohamed Ibrahim (2005): Encyclopedia of sports training parameterstheory and practice - for planning karate training programs, Dar ELMaaref, Alexandria

6. Ahmed Mahmoud Mohamed Ibrahim, Amr Allah Ahmed El-Bisatti (1999): Effect of directing training loads according to bio-rhythm pattern on some functional indications and skills performance level of karate player, theories and applications, Faculty of Physical Education for Men, the second number and Thirty, Alexandria University, Alexandria. 
7. Ahmed Mahmoud Mohamed Ibrahim(2000): differentiation and relative contribution of some biological and physical indications for distinctive and indistinctive Kata players as selection and classification parameter in karate, scientific journal Physical Education and Sports, Physical Faculty of physical Education for Girls, No. 18 , Alexandria University

8. Ahmed Mahmoud Mohamed Ibrahim, Atef Mohamed Abaza (2005): scientific and applied basics for planning training programs for "kata" in karate, Dae EL Maaref, Alexandria>

9. Tamer Saleh Shehadeh El-Absi (2007): biorhythm and motor parameter as a basis for training program development for 3000 meters running for juniors in Palestine, Master thesis, Faculty of Physical Education for girls, Alexandria University.

10. Hossam Eddin Abdel Razek Hawari Hassan (1999): Relationship between bio-rhythm and pattern of physical abilities and some functional characteristics and achievement player of wrestling players, Master Thesis, Faculty of Physical Education for Men in Cairo, Helwan University.

11. Doaa Ibrahim El-Gamal (2008): Impact of a training program according to physical biorhythm curve on some biological variables and 25 meters freestyle swimming time, Master Thesis, Faculty of Physical Education for Girls, Alexandria University.

12. Ali Fahmi El Beck (1990): Biologic rhythm and athletic achievement, published research Scientific Conference - Physical Education role in solving contemporary problems siminar, Faculty of Physical Education for Men, Zagazig University.

13. Ali Fahmi Elbek and Mohammed Sabri Omar (1994): bio-rhythm and athletic achievement, Dar ElMaaref, Alexandria.

14. Ali Abdul Azzazi (1992): Effect of day periods on flexibility factor of for youth 18-20 years old, published research, Journal of the theories and applications, Faculty of Physical Education for Men in Alexandria, second issue, January.

15. Ghada Mohamed (1996): Sports training programs planning according to bio-rhythm dynamics and its effect on a 100 meter sprint time of preparatory school pupils in Alexandria, $\mathrm{PhD}$ thesis, Faculty of Physical Education for Girls, Tanta University.

16. Fadel Soliman Sherida Khalidi (1990): Biological rhythm and its impact on athletic level achievement, fifth sports medicine course for technicians, Arab Saudi Union of Sports Medicine, Saudi Arabia.

17. Mohammad Hassan Allawi (2002): psychomotor parameters in light of technological development and information revolution, International Scientific Conference, Strategies of talent athletes selection and preparation in light of technological development and the information revolution in the period from October 30 to November 1, Faculty of Physical Education for Men Alexandria University.

18. Mohamed Sobhi Hassanein (2002): Athlete selection, International Scientific Conference, Strategies of talent athletes selection and preparation in light of technological development and the information revolution in the period from October 30 to November 1, Faculty of Physical Education for Men Alexandria University.

19. Mohammed Sabri Omar(1991): differentiation analysis of high/normal level female swimmers in crawl swimming physical measurements, theories and applications journal, Faculty of Physical Education for Men, No.11, Alexandria University.

20. Mohammed Sabri Omar (2002): Scientific research trends in physical and biological parameters for athlete selection in light of technological development and information revolution, International Scientific Conference, Strategies of talent athletes selection and preparation in light of technological development and the information revolution in the period from October 30 to November 1, Faculty of Physical Education for Men Alexandria University.

21. Yossuf Dahab Ali (1993): biological rhythm for athlete body as an indicator of academic achievement and physical activity rates, Journal of Scientific Conference: gymnastics, exercises and physical fitness for all, " rehabilitation and progression" Faculty of Physical Education for Men, Alexandria University 
22. Yosuf Dahab Ali (2002): physiological reserve, selection processes and functional efficiency standardization, International Scientific Conference, Strategies of talent athletes selection and preparation in light of technological development and the information revolution in the period from October 30 to November 1, Faculty of Physical Education for Men Alexandria University.

23. Yosuf Dahab Ali, Mohammed Jaber Briqaa, Ahmed Mohamed Ibrahim (1993): Arabization and standardization of Ostbreg scale to determine bio-rhythm style, scientific journal of Physical Education and Sports, Conference researches: future vision of physical education and sports in Arab world, Faculty of Physical Education for Men, Helwan University.

24. Armstrong, L (1998):Desynchronization of biological rhythms in athletes. Jet lag National strength and conditioning Association Journal,
Vol.10, part 6, Jehue, R., Street, D., Huizenga, R. (1993):Effect of time zone and game time changes on team performance, Med. Sci. Sports exercise, vol 26 part 1, U.S.A, Kouted A.kisy (1995): seasonal variegation in fitness parameters in competitive Athletes sports medicine " Kuckland N.Z.England "

25. Kramer, W.etal (1998): Biorhythmic Influences on Functional capacity of Human muscle and physiological responses Mad : Sci sport , sep ,30(9)1399-407

26. Philips, B. (1994): Circadiam rhythms and muscular strength performance "Mountain wiew, calif, trach and dield mens.

27. Reilly, T. (1990):"Human circadian rhythm \& Exercise" center for sfosts and elercises, levespool, England, 1990

28. Robin, L. (2005):Complete shotokan karate, charless \& Tuttle. Co., U.S.A. 